\title{
Editorial \\ Publisher's Note: We Changed Page Numbers to Article Numbers for Articles Published in Audiology Research Volume 1-Volume 10, Issue 1
}

\section{Audiology Research Editorial Office}

MDPI AG, St. Alban-Anlage 66, 4052 Basel, Switzerland; audiolres@mdpi.com

check for updates

Citation: Audiology Research Editorial Office. Publisher's Note: We Changed Page Numbers to Article Numbers for Articles Published in Audiology Research Volume 1-Volume 10, Issue 1. Audiol. Res. 2022, 12, 96-98. https://doi.org/10.3390/ audiolres12020012

Received: 22 February 2022 Accepted: 22 February 2022 Published: 23 February 2022

Publisher's Note: MDPI stays neutral with regard to jurisdictional claims in published maps and institutional affiliations.

Copyright: (C) 2022 by the author. Licensee MDPI, Basel, Switzerland. This article is an open access article distributed under the terms and conditions of the Creative Commons Attribution (CC BY) license (https:// creativecommons.org/licenses/by/ $4.0 /)$.
Audiology Research [1] was published by PAGEPress from Volume 1 (2011) to Volume 10, Issue 1 (2020). From Volume 10, Issue 2 (2020), Audiology Research has been published by MDPI.

Previous articles in Volumes 1-10, published by PAGEPress in Open Access under a CC-BY (or CC-BY-NC-ND) license, are now hosted by MDPI on mdpi.com as a courtesy and upon agreement with PAGEPress.

To standardize the metadata format of all previous articles, MDPI republished 125 articles in Volume 1-Volume 10, Issue 1, with article numbers replacing page numbers (Table 1).

Table 1. MDPI has changed the page numbers to article numbers for 125 articles in Volumes 1-10.

\begin{tabular}{ccc}
\hline DOI & Previous Page Number & Current Article Number \\
\hline 10.4081/audiores.2011.e1 & 1 & e1 \\
10.4081/audiores.2011.e2 & $3-5$ & e2 \\
10.4081/audiores.2011.e3 & $6-8$ & e3 \\
10.4081/audiores.2011.e4 & $9-15$ & e4 \\
10.4081/audiores.2011.e5 & $16-18$ & e5 \\
10.4081/audiores.2011.e6 & $19-21$ & e6 \\
10.4081/audiores.2011.e7 & $22-24$ & e7 \\
10.4081/audiores.2011.e8 & $25-27$ & e8 \\
10.4081/audiores.2011.e9 & $28-33$ & e9 \\
10.4081/audiores.2011.e10 & $34-39$ & e10 \\
10.4081/audiores.2011.e11 & $40-41$ & e11 \\
10.4081/audiores.2011.e12 & $42-48$ & e12 \\
10.4081/audiores.2011.e13 & $49-54$ & e13 \\
10.4081/audiores.2011.e14 & $55-57$ & e14 \\
10.4081/audiores.2011.e15 & $58-60$ & e15 \\
10.4081/audiores.2011.e16 & $61-63$ & e16 \\
10.4081/audiores.2011.e17 & $64-68$ & e17 \\
10.4081/audiores.2011.e18 & $69-70$ & e18 \\
10.4081/audiores.2011.e19 & $71-73$ & e19 \\
10.4081/audiores.2011.e20 & $74-78$ & e20 \\
10.4081/audiores.2011.e21 & $79-81$ & e21 \\
10.4081/audiores.2011.e22 & $82-85$ & e22 \\
10.4081/audiores.2011.e23 & $86-87$ & e23 \\
10.4081/audiores.2011.e24 & $88-90$ & e24 \\
10.4081/audiores.2011.e25 & $91-96$ & e25 \\
10.4081/audiores.2011.e26 & $97-110$ & e26 \\
10.4081/audiores.2011.e27 & $1-8$ & e27 \\
10.4081/audiores.2011.e28 & $9-12$ & e28 \\
10.4081/audiores.2011.e29 & $13-17$ & e30 \\
10.4081/audiores.2011.e30 & $18-22$ & \\
\hline & & a 29 \\
\hline
\end{tabular}


Table 1. Cont.

\begin{tabular}{|c|c|c|}
\hline DOI & Previous Page Number & Current Article Number \\
\hline 10.4081/audiores.2011.e31 & $23-29$ & e31 \\
\hline 10.4081/audiores.2011.ed & 1 & ed \\
\hline 10.4081/audiores.2012.e1 & $1-3$ & e1 \\
\hline 10.4081/audiores.2012.e2 & $4-7$ & e2 \\
\hline 10.4081/audiores.2012.e3 & $8-11$ & e3 \\
\hline 10.4081/audiores.2012.e4 & $12-16$ & e4 \\
\hline 10.4081/audiores.2012.e5 & $17-24$ & e5 \\
\hline 10.4081/audiores.2012.e6 & $25-29$ & e6 \\
\hline 10.4081/audiores.2012.e7 & $30-38$ & e7 \\
\hline 10.4081/audiores.2012.e8 & $39-42$ & e8 \\
\hline 10.4081/audiores.2012.e9 & $43-47$ & e9 \\
\hline 10.4081/audiores.2012.e11 & $57-63$ & e11 \\
\hline 10.4081/audiores.2012.e12 & 64 & e12 \\
\hline 10.4081/audiores.2012.e13 & $65-76$ & e13 \\
\hline 10.4081/audiores.2012.e14 & $77-85$ & e14 \\
\hline 10.4081/audiores.2012.e15 & $86-93$ & e15 \\
\hline 10.4081/audiores.2013.e1 & $1-9$ & e1 \\
\hline 10.4081/audiores.2013.e2 & $10-15$ & e2 \\
\hline 10.4081/audiores.2013.e3 & $16-25$ & e3 \\
\hline 10.4081/audiores.2013.e4 & $26-31$ & e4 \\
\hline 10.4081/audiores.2013.e5 & $32-41$ & e5 \\
\hline 10.4081/audiores.2013.e6 & $42-47$ & e6 \\
\hline 10.4081/audiores.2013.e7 & $48-51$ & e7 \\
\hline 10.4081/audiores.2013.e8 & $52-56$ & e8 \\
\hline 10.4081/audiores.2013.e9 & $57-62$ & e9 \\
\hline 10.4081/audiores.2014.80 & $36-39$ & 80 \\
\hline 10.4081/audiores.2014.88 & $14-20$ & 88 \\
\hline 10.4081/audiores.2014.89 & $9-13$ & 89 \\
\hline 10.4081/audiores.2014.91 & $5-8$ & 91 \\
\hline 10.4081/audiores.2014.93 & $1-4$ & 93 \\
\hline 10.4081/audiores.2014.99 & $56-60$ & 99 \\
\hline 10.4081/audiores.2014.101 & $28-35$ & 101 \\
\hline 10.4081/audiores.2014.102 & $40-45$ & 102 \\
\hline 10.4081/audiores.2014.106 & $21-27$ & 106 \\
\hline 10.4081/audiores.2014.108 & $52-55$ & 108 \\
\hline 10.4081/audiores.2014.110 & $46-51$ & 110 \\
\hline 10.4081/audiores.2015.105 & $1-4$ & 105 \\
\hline 10.4081/audiores.2015.111 & $5-8$ & 111 \\
\hline 10.4081/audiores.2015.113 & $21-29$ & 113 \\
\hline 10.4081/audiores.2015.116 & $44-49$ & 116 \\
\hline 10.4081/audiores.2015.117 & $9-19$ & 117 \\
\hline 10.4081/audiores.2015.120 & $30-33$ & 120 \\
\hline 10.4081/audiores.2015.121 & $65-68$ & 121 \\
\hline 10.4081/audiores.2015.128 & $34-37$ & 128 \\
\hline 10.4081/audiores.2015.130 & $38-43$ & 130 \\
\hline 10.4081/audiores.2015.132 & $69-75$ & 132 \\
\hline 10.4081/audiores.2015.133 & $50-56$ & 133 \\
\hline 10.4081/audiores.2015.135 & $57-64$ & 135 \\
\hline 10.4081/audiores.2015.136 & $80-83$ & 136 \\
\hline 10.4081/audiores.2015.139 & $76-79$ & 139 \\
\hline 10.4081/audiores.2016.137 & $1-5$ & 137 \\
\hline 10.4081/audiores.2016.140 & $11-13$ & 140 \\
\hline 10.4081/audiores.2016.146 & $6-10$ & 146 \\
\hline 10.4081/audiores.2016.147 & $14-16$ & 147 \\
\hline 10.4081/audiores.2016.151 & $17-21$ & 151 \\
\hline 10.4081/audiores.2016.152 & $44-48$ & 152 \\
\hline 10.4081/audiores.2016.153 & $22-27$ & 153 \\
\hline 10.4081/audiores.2016.154 & $28-35$ & 154 \\
\hline
\end{tabular}


Table 1. Cont.

\begin{tabular}{|c|c|c|}
\hline DOI & Previous Page Number & Current Article Number \\
\hline 10.4081/audiores.2016.158 & $40-43$ & 158 \\
\hline 10.4081/audiores.2016.159 & $49-57$ & 159 \\
\hline 10.4081 /audiores.2016.160 & $58-63$ & 160 \\
\hline 10.4081 /audiores.2017.157 & $1-5$ & 157 \\
\hline 10.4081/audiores.2017.161 & $15-22$ & 161 \\
\hline 10.4081/audiores.2017.162 & $6-9$ & 162 \\
\hline 10.4081/audiores.2016.163 & $36-39$ & 163 \\
\hline 10.4081/audiores.2017.165 & $23-26$ & 165 \\
\hline 10.4081/audiores.2017.168 & $10-14$ & 168 \\
\hline 10.4081/audiores.2017.176 & $42-46$ & 176 \\
\hline 10.4081/audiores.2017.178 & $31-37$ & 178 \\
\hline 10.4081/audiores.2017.181 & $38-41$ & 181 \\
\hline 10.4081/audiores.2017.182 & $61-66$ & 182 \\
\hline 10.4081 /audiores.2017.185 & $51-55$ & 185 \\
\hline 10.4081/audiores.2017.187 & $47-50$ & 187 \\
\hline 10.4081/audiores.2017.189 & $56-60$ & 189 \\
\hline 10.4081 /audiores.2017.190 & $67-70$ & 190 \\
\hline 10.4081/audiores.2018.194 & $1-4$ & 194 \\
\hline 10.4081/audiores.2018.198 & $9-12$ & 198 \\
\hline 10.4081/audiores.2018.200 & $5-8$ & 200 \\
\hline 10.4081 /audiores.2018.204 & $16-23$ & 204 \\
\hline 10.4081/audiores.2018.206 & $24-26$ & 206 \\
\hline 10.4081/audiores.2018.212 & $34-36$ & 212 \\
\hline 10.4081/audiores.2018.214 & $27-33$ & 214 \\
\hline 10.4081/audiores.2018.215 & $37-43$ & 215 \\
\hline 10.4081 /audiores.2018.216 & $44-53$ & 216 \\
\hline 10.4081/audiores.2019.217 & $1-5$ & 217 \\
\hline 10.4081/audiores.2019.219 & $6-9$ & 219 \\
\hline 10.4081/audiores.2019.222 & $10-13$ & 222 \\
\hline 10.4081/audiores.2019.223 & $14-22$ & 223 \\
\hline 10.4081/audiores.2019.228 & $23-26$ & 228 \\
\hline 10.4081/audiores.2019.230 & $27-32$ & 230 \\
\hline 10.4081/audiores.2019.231 & $33-37$ & 231 \\
\hline 10.4081 /audiores.2020.232 & $12-15$ & 232 \\
\hline 10.4081 /audiores.2020.233 & $1-5$ & 233 \\
\hline 10.4081/audiores.2020.234 & $16-20$ & 234 \\
\hline 10.4081/audiores.2020.236 & $6-11$ & 236 \\
\hline
\end{tabular}

Conflicts of Interest: The author declares no conflict of interest.

\section{Reference}

1. Audiology Research Homepage. Available online: https://www.mdpi.com/journal/audiolres (accessed on 10 February 2022). 